\title{
Non-associative slave-boson decomposition
}

\author{
Vladimir DZHUNUSHALIEV ${ }^{1}$ \\ Department of Physical and Microelectronical Engineering, Kyrgyz-Russian Slavic University, \\ Kievskaya Str. 44, 720021 Bishkek, Kyrgyz Republic
}

E-mail:dzhun@krsu.edu.kg

\begin{abstract}
An operator constraint for a slave-boson decomposition in $t$ - $J$ model of high temperature superconductivity is considered. It is shown that the constraint can be resolved by introducing a non-associative operator. In this case the constraint is an antiassociative generating relation of a new algebra. Similar constraint is offered for splitting the gluon.
\end{abstract}

2000 MSC: 81Rxx, 17D05

\section{Introduction}

Everybody knows that the algebra of non-perturbative operators in quantum theory exists but nobody knows its exact form. In this paper the idea is discussed that the constraint (2.2) in $t-J$ model of high-temperature superconductivity is a new generating relation for an algebra of operators the product of which gives us the electron operator. On the perturbative level the algebra of quantum fields is defined by canonical (anti)commutative relations. The algebra of non-perturbative operators should be more complicated and should be generated not only by canonical (annti)commutative relations but should exist other generating relations as well. In this paper we discuss the idea that the constraint (2.2) is an antiassociator in a non-associative algebra of quantum non-perturbative operators.

In $[14,10,11]$, the authors apply non-associative algebras to physics; the topics ranging from algebras of observables in quantum mechanics, to angular momentum and octonions, division algebras, triple-linear products and Yang - Baxter equations are covered. The non-associative gauge theoretic reformulation of Einstein's general relativity theory is also discussed. In [1] one can also find the review of mathematical definitions and physical applications for the octonions.

\section{Operator properties of $t-J$ model}

It is widely believed that the low energy physics of high temperature (High- $\mathrm{T}_{c}$ ) cuprates (for a review see [9]) is described in terms of $t$ - $J$ type model, which is given [8] by

$$
H=\sum_{i, j} J\left(S_{i} \cdot S_{j}-\frac{1}{4} n_{i} n_{j}\right)-\sum_{i, j} t_{i j}\left(c_{i \sigma}^{\dagger} c_{j \sigma}+\text { H.c. }\right)
$$

where $t_{i j}=t, t^{\prime}, t^{\prime \prime}$ for the nearest, second nearest and 3rd nearest neighbor pairs, respectively. The effect of the strong Coulomb repulsion is represented by the fact that the electron operators $c_{i \sigma}^{\dagger}$ and $c_{i \sigma}$ are the projected ones, where the double occupation is forbidden. This can be written as the inequality $\sum_{\sigma} c_{i \sigma}^{\dagger} c_{i \sigma} \leq 1$, which is very difficult to handle. A powerful method to treat this constraint is the so-called slave-boson method $[2,4]$. In this approach the electron operator is represented as

$$
c_{i \sigma}^{\dagger}=f_{i \sigma}^{\dagger} b_{i}
$$

\footnotetext{
${ }^{1}$ Senior Associate of the Abdus Salam ICTP
} 
where $f_{i \sigma}^{\dagger}, f_{i \sigma}$ are the fermion operators, while $b_{i}$ is the slave-boson operator. This representation together with the constraint

$$
f_{i \uparrow}^{\dagger} f_{i \uparrow}+f_{i \downarrow}^{\dagger} f_{i \downarrow}+b_{i}^{\dagger} b_{i}=1
$$

reproduces all the algebra of the electron operators. The physical meaning of the operators $f$ and $b$ is unclear: do these fields exist or not?

In this paper we would like to show that the constraint (2.2) can be considered as a generating relation for a new algebra of operators from which the electron operator is constructed.

\section{A simple physical consideration}

At this stage we ignore all indexes in the constraint (2.2). In this case Eq. (2.2) has the form

$$
f^{\dagger} f+b^{\dagger} b=1
$$

Now we want to compare this equation with the one of generating relations in a non-associative algebra proposed in $[5,6]$. Let us assume that there exists a non-associative algebra $G$. The algebra $G$ is generated with associators and antiassociators (shortly speaking ( \pm )associators). One of the (-)associators has the form

$$
\left(Q_{1} Q_{2}\right) Q_{3}+Q_{1}\left(Q_{2} Q_{3}\right)=(\text { something })
$$

where $Q_{i}, i=1,2,3$ are non-associative operators and it is the antiassociator (5.2) from Section 5.2. In the simplest case (something) $=1$. Let us compare both Eq's (2.2) and (3.2). It is easy to see that they are identical if we assign

$$
f^{\dagger}=Q_{1} Q_{2}, \quad f=Q_{3}, \quad b^{\dagger}=Q_{1}, \quad b=Q_{2} Q_{3}
$$

Immediately we can see that by using Eq's (3.3), Eq. (3.2) can be rewritten in the following way:

$$
\left(b^{\dagger} Q_{2}\right) f+b^{\dagger}\left(Q_{2} f\right)=1
$$

here for the simplicity we assume that r.h.s of (3.2) is unity. Eq. (3.4) tells us that the constraint (3.1) can be resolved in a non-associative algebra by the introduction of a nonassociative operator $Q_{2}$.

Thus the idea presented here is that the slave-boson decomposition is nothing else than the decomposition of an associative operator on non-associative operators. Such non-associative algebra should have an associative subalgebra with the elements $c$ given as

$$
c=f^{\dagger} b
$$

where $f$ and $b$ are non-associative operators. In other words, in a non-associative algebra $G$ the observables $c$ have the slave-boson decomposition (3.5) where non-associative operators $f$ and $b$ are unobservables quantities.

Now we can restore the spin index $\sigma$ and write

$$
f_{\sigma}^{\dagger}=Q_{1} Q_{2 \sigma}, \quad f_{\sigma}=Q_{3 \sigma}, \quad b^{\dagger}=Q_{1}, \quad b=\sum_{\sigma} Q_{2 \sigma} Q_{3 \sigma}
$$

where $\sigma=\{\uparrow, \downarrow\}$ is the spin index. In this case the (-)associator (3.2) has the form

$$
\sum_{\sigma}\left(Q_{1} Q_{2 \sigma}\right) Q_{3 \sigma}+Q_{1}\left(\sum_{\sigma} Q_{2 \sigma} Q_{3 \sigma}\right)=(\text { something })
$$


Analogously to Eq. (3.4) the non-associative solution of (2.2) is

$$
\sum_{\sigma}\left(b^{\dagger} Q_{2 \sigma}\right) f_{\sigma}+b^{\dagger}\left(\sum_{\sigma} Q_{2 \sigma} f_{\sigma}\right)=\text { (something) }
$$

where $Q_{2 \sigma}$ is unknown non-associative operator.

The problem of such interpretation of the constraint (2.2) is evident: does there exist a non-associative algebra with (-)associators (3.2) and (3.6)?

\section{$4 \quad$ Splitting the gluon?}

The title of this section is the same as the title of Ref. [12] where it is shown that there exists the decomposition of gluon in Yang-Mills gauge theory similar to the slave-boson decomposition in High- $\mathrm{T}_{c}$ superconductivity. In Ref. [3] the similar construction (spin-charge separation) for the gauge boson is offered as well. The physical ground is that in both cases we are dealing with strong interactions: between electrons in High- $\mathrm{T}_{c}$ superconductivity and gauge bosons in quantum chromodynamics.

In Ref. [12] the slave-boson decomposition of the $S U(2)$ gauge field $A_{\mu}^{a}$ ( $a=1,2,3$ and $\mu=0,1,2,3)$ proceeds as follows [7, 13]: at first the diagonal Cartan component $A_{\mu}^{3}=A_{\mu}$ from the off-diagonal components $A_{\mu}^{1,2}$ is separated, and combined the latter into the complex field $W_{\mu}=A_{\mu}^{1}+i A_{\mu}^{2}$. Then a complex vector field $\vec{e}_{\mu}$ with the properties

$$
\vec{e}_{\mu} \vec{e}_{\mu}=0 \quad \text { and } \quad \vec{e}_{\mu} \vec{e}_{\mu}^{*}=1
$$

is introduced; two spinless complex scalar fields $\psi_{1}$ and $\psi_{2}$ are introduced as well. The ensuing decomposition of $W_{\mu}$ is [7]

$$
W_{\mu}=A_{\mu}^{1}+i A_{\mu}^{2}=\psi_{1} \vec{e}_{\mu}+\psi_{2}^{*} \vec{e}_{\mu}^{*}
$$

This is a direct analogue of Eq. (2.2), a decomposition of $W_{\mu}$ into spinless bosonic scalars $\psi_{1,2}$ which describe the gluonic holons that carry the color charge of the $W_{\mu}$, and a color-neutral spin-one vector $\vec{e}_{\mu}$ which is the gluonic spinon that carries the statistical spin degrees of freedom of $W_{\mu}$.

In Ref. [3] the spin-charge separation of $\mathrm{SU}(2)$ gauge potential is given by a little another way

$$
A_{\mu}^{a}=e_{\mu}^{i} \Phi^{i a}
$$

where $a=1,2,3$ is the $\mathrm{SU}(2)$ color index; $i=1,2,3$ is an inner index and $\mu=0,1,2,3$ is the Lorentzian index. The decompositions (4.1) and (4.2) are the decompositions of the classical fields. If we trust the quantum slave-boson decomposition for strongly interacting electrons in High- $\mathrm{T}_{c}$ superconductivity then we can apply this idea for the strongly interacting $\mathrm{SU}(3)$ gauge potential. In this case the non-perturbative operator $\hat{A}_{\mu}^{B}$ can be decomposed in the following way

$$
\hat{A}_{\mu}^{B}=\hat{e}_{\mu}^{i} \hat{\Phi}^{i B}
$$

where we follow to the decomposition (4.2); $B=1, \cdots, 8$ is the $\mathrm{SU}(3)$ color index. Following to the slave-boson idea we assume that there is the constraint

$$
\sum_{i, \mu} \hat{e}_{\mu}^{\dagger i} \hat{e}^{i \mu}+\sum_{i, B} \hat{\Phi}^{\dagger i B} \hat{\Phi}^{i B}=1
$$


Analogously to (2.2) the resolution of this constraint is a (-) associator

$$
\sum_{i, j, \mu, B}\left(\hat{\Phi}^{\dagger j B} \hat{Q}_{\mu}^{j i B}\right) \hat{e}^{i \mu}+\sum_{i, j, \mu, B} \hat{\Phi}^{\dagger i B}\left(\hat{Q}_{\mu}^{i j B} \hat{e}^{j \mu}\right)=1
$$

with

$$
\hat{e}_{\mu}^{\dagger i}=\sum_{j, B}\left(\hat{\Phi}^{\dagger j B} \hat{Q}_{\mu}^{j i B}\right), \quad \hat{\Phi}^{i B}=\sum_{j, \mu}\left(\hat{Q}_{\mu}^{i j B} \hat{e}^{j \mu}\right)
$$

where $\hat{Q}_{\mu}^{i j B}$ is an unknown non-associative operator.

In the next section we will present a non-associative algebra where $( \pm)$ associators are given on the level of the product of three operators.

\section{A non-associative algebra}

In this section we will follow [5]. At first we would like to note that: (a) non-associative algebras proposed in $[5,6]$ are the operator generalization of the octonions; (b) the full definition of this algebra is jet unknown and here we give the $( \pm)$ associators for the product of three operators only.

\subsection{Octonions}

In this subsection we give a very short description what are the octonion numbers. Let splitoctonion numbers are denoted as $\tilde{q}_{i}$ and $\tilde{Q}_{j}$. In Table 1 we present the multiplication rule of the split-octonion units $\tilde{q}_{i}, \tilde{Q}_{j}$ and $I$.

\begin{tabular}{|c|c|c|c|c|c|c|c|}
\hline & $\tilde{q}_{1}$ & $\tilde{q}_{2}$ & $\tilde{q}_{3}$ & $\tilde{Q}_{1}$ & $\tilde{Q}_{2}$ & $\tilde{Q}_{3}$ & $I$ \\
\hline$\tilde{q}_{1}$ & -1 & $\tilde{q}_{3}$ & $-\tilde{q}_{2}$ & $-I$ & $\tilde{Q}_{3}$ & $-\tilde{Q}_{2}$ & $\tilde{Q}_{1}$ \\
\hline$\tilde{q}_{2}$ & $-\tilde{q}_{3}$ & -1 & $\tilde{q}_{1}$ & $-\tilde{Q}_{3}$ & $-I$ & $\tilde{Q}_{1}$ & $\tilde{Q}_{2}$ \\
\hline$\tilde{q}_{3}$ & $\tilde{q}_{2}$ & $-\tilde{q}_{1}$ & -1 & $\tilde{Q}_{2}$ & $-\tilde{Q}_{1}$ & $-I$ & $\tilde{Q}_{3}$ \\
\hline$\tilde{Q}_{1}$ & $I$ & $\tilde{Q}_{3}$ & $-\tilde{Q}_{2}$ & 1 & $-\tilde{q}_{3}$ & $\tilde{q}_{2}$ & $\tilde{q}_{1}$ \\
\hline$\tilde{Q}_{2}$ & $-\tilde{Q}_{3}$ & $I$ & $\tilde{Q}_{1}$ & $\tilde{q}_{3}$ & 1 & $-\tilde{q}_{1}$ & $\tilde{q}_{2}$ \\
\hline$\tilde{Q}_{3}$ & $\tilde{Q}_{2}$ & $-\tilde{Q}_{1}$ & $I$ & $-\tilde{q}_{2}$ & $\tilde{q}_{1}$ & 1 & $\tilde{q}_{3}$ \\
\hline$I$ & $-\tilde{Q}_{1}$ & $-\tilde{Q}_{2}$ & $-\tilde{Q}_{3}$ & $-\tilde{q}_{1}$ & $-\tilde{q}_{2}$ & $-\tilde{q}_{3}$ & 1 \\
\hline
\end{tabular}

Table 1. The split-octonions multiplication table.

\subsection{Quantum ( \pm )associators}

In this section we present the quantum $( \pm$ )associators for the product of three operators. The anticommutators are

$$
\left\{q_{i} q_{j}\right\}_{+}=0, \quad\left\{q_{i} Q_{j}\right\}_{+}=0, \quad\left\{Q_{i} Q_{j}\right\}_{+}=0, \quad\left\{q_{i} Q_{i}\right\}_{+}=0
$$

The quantum $( \pm$ )associators with different indices $m \neq n, n \neq p, p \neq m$ are

$$
\begin{aligned}
\left\{q_{m}, q_{n}, q_{p}\right\}_{-} & =\left(q_{m} q_{n}\right) q_{p}-q_{m}\left(q_{n} q_{p}\right)=0 \\
\left\{Q_{m}, Q_{n}, Q_{p}\right\}_{+} & =\left(Q_{m} Q_{n}\right) Q_{p}+Q_{m}\left(Q_{n} Q_{p}\right)=\epsilon_{m n p} \mathcal{H}_{3,1} \\
\left\{q_{m}, Q_{n}, q_{p}\right\}_{+} & =\left(q_{m} Q_{n}\right) q_{p}+q_{m}\left(Q_{n} q_{p}\right)=\epsilon_{m n p} \mathcal{H}_{3,2}
\end{aligned}
$$




$$
\begin{aligned}
\left\{Q_{m}, q_{n}, Q_{p}\right\}_{-} & =\left(Q_{m} q_{n}\right) Q_{p}-Q_{m}\left(q_{n} Q_{p}\right)=\epsilon_{m n p} \mathcal{H}_{3,3} \\
\left\{q_{m}, q_{n}, Q_{p}\right\}_{-} & =\left(q_{m} q_{n}\right) Q_{p}-q_{m}\left(q_{n} Q_{p}\right)=\epsilon_{m n p} \mathcal{H}_{3,4} \\
\left\{Q_{m}, q_{n}, q_{p}\right\}_{-} & =\left(Q_{m} q_{n}\right) q_{p}-Q_{m}\left(q_{n} q_{p}\right)=\epsilon_{m n p} \mathcal{H}_{3,5} \\
\left\{q_{m}, Q_{n}, Q_{p}\right\}_{-} & =\left(q_{m} Q_{n}\right) Q_{p}-q_{m}\left(Q_{n} Q_{p}\right)=\epsilon_{m n p} \mathcal{H}_{3,6} \\
\left\{Q_{m}, Q_{n}, q_{p}\right\}_{-} & =\left(Q_{m} Q_{n}\right) q_{p}-Q_{m}\left(Q_{n} q_{p}\right)=\epsilon_{m n p} \mathcal{H}_{3,7}
\end{aligned}
$$

where $\mathcal{H}_{3, i}$ are operators (but may be they are numbers); Eq. (5.1) means that the quaternioniclike subalgebra spanned on $q_{1}, q_{2}, q_{3}$ is the associative algebra. The quantum antiassociators for the product of three operators, such as $q(Q q)$ or $Q(q Q)$, having two different indices $m \neq n$ are

$$
\begin{aligned}
\left\{q_{m}, Q_{n}, q_{n}\right\}_{+} & =q_{m}\left(Q_{n} q_{n}\right)+\left(q_{m} Q_{n}\right) q_{n}=\mathcal{H}_{3,8}(m, n) \\
\left\{q_{n}, Q_{n}, q_{m}\right\}_{+} & =q_{n}\left(Q_{n} q_{m}\right)+\left(q_{n} Q_{n}\right) q_{m}=\mathcal{H}_{3,9}(m, n) \\
\left\{Q_{m}, q_{n}, Q_{n}\right\}_{+} & =Q_{m}\left(q_{n} Q_{n}\right)+\left(Q_{m} q_{n}\right) Q_{n}=\mathcal{H}_{3,10}(m, n) \\
\left\{Q_{n}, q_{n}, Q_{m}\right\}_{+} & =Q_{n}\left(q_{n} Q_{m}\right)+\left(Q_{n} q_{n}\right) Q_{m}=\mathcal{H}_{3,11}(m, n)
\end{aligned}
$$

The quantum associators, such as $q(Q Q)$ or $Q(q q)$, and with two different indices $m \neq n$ are

$$
\begin{aligned}
& \left\{q_{m}, Q_{m}, Q_{n}\right\}_{-}=\left(q_{m} Q_{m}\right) Q_{n}-q_{m}\left(Q_{m} Q_{n}\right)=\mathcal{H}_{3,12}(m, n) \\
& \left\{q_{m}, Q_{n}, Q_{m}\right\}_{-}=\left(q_{m} Q_{n}\right) Q_{m}-q_{m}\left(Q_{n} Q_{m}\right)=\mathcal{H}_{3,13}(m, n) \\
& \left\{Q_{n}, Q_{m}, q_{m}\right\}_{-}=\left(Q_{n} Q_{m}\right) q_{m}-Q_{n}\left(Q_{m} q_{m}\right)=\mathcal{H}_{3,14}(m, n) \\
& \left\{Q_{m}, Q_{n}, q_{m}\right\}_{-}=\left(Q_{m} Q_{n}\right) q_{m}-Q_{m}\left(Q_{n} q_{m}\right)=\mathcal{H}_{3,15}(m, n) \\
& \left\{Q_{m}, q_{m}, q_{n}\right\}_{-}=\left(Q_{m} q_{m}\right) q_{n}-Q_{m}\left(q_{m} q_{n}\right)=\mathcal{H}_{3,16}(m, n) \\
& \left\{Q_{m}, q_{n}, q_{m}\right\}_{-}=\left(Q_{m} q_{n}\right) q_{m}-Q_{m}\left(q_{n} q_{m}\right)=\mathcal{H}_{3,17}(m, n) \\
& \left\{q_{n}, q_{m}, Q_{m}\right\}_{-}=\left(q_{n} q_{m}\right) Q_{m}-q_{n}\left(q_{m} Q_{m}\right)=\mathcal{H}_{3,18}(m, n) \\
& \left\{q_{m}, q_{n}, Q_{m}\right\}_{-}=\left(q_{m} q_{n}\right) Q_{m}-q_{m}\left(q_{n} Q_{m}\right)=\mathcal{H}_{3,19}(m, n)
\end{aligned}
$$

where $\mathcal{H}_{3, i}(m, n)$ are operators (but may be they are numbers). The alternativity properties are

$$
\begin{aligned}
& \left\{q_{n}, q_{n}, Q_{m}\right\}_{-}=\left(q_{n} q_{n}\right) Q_{m}-q_{n}\left(q_{n} Q_{m}\right)=0 \\
& \left\{q_{n}, Q_{m}, q_{n}\right\}_{-}=\left(q_{n} Q_{m}\right) q_{n}-q_{n}\left(Q_{m} q_{n}\right)=0 \\
& \left\{Q_{m}, q_{n}, q_{n}\right\}_{-}=\left(Q_{m} q_{n}\right) q_{n}-Q_{m}\left(q_{n} q_{n}\right)=0
\end{aligned}
$$

\subsection{Self-consistency of quantum $( \pm)$ associators}

The self-consistency of the $( \pm$ )associators for the product of three operators can be proved according to the commutative diagram (5.3). For this we permute the first and third factors in the product $a(b c)$ by following the commutative diagram

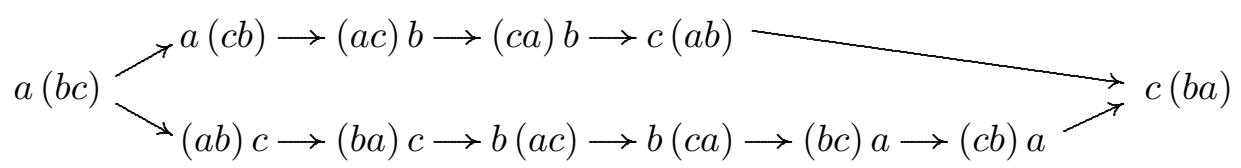

In $[5,6]$ it is shown that the $( \pm)$ associators with the product of three operators are self-consistent. On this level it is impossible to define operators $\mathcal{H}_{3, i}$.

\section{Outlook}

In this paper we have shown that the constraint (2.2) can be resolved by an unexpected manner: the constraint is a generating relation for a new non-associative algebra. The bilinear 
combination of operators of this algebra make up an operator of an observable physical quantity. Probably: (a) such decomposition can be done only in the case if these physical quantities have strong interaction; (b) there exists an associative subalgebra of the above-mentioned nonassociative algebra where associative operators can be decomposed by non-associative operators following to the slave-boson way. In [6] such observable physical quantities are called white (colorless) operators. One can say that this hypothesized decomposition of an associative operator on non-associative operators (similar Eq's (2.1) and (4.3)) is in some sense the generalization of quark confinement hypothesis: in both cases we have observable physical quantities which are build from unobservable quantities.

\section{Acknowledgements}

The author thanks D. Ebert, M. Mueller-Preussker and the other colleagues of the Particle Theory Group of the Humboldt University for kind hospitality. This work has been supported by DAAD.

\section{References}

[1] J. C. Baez. The Octonions. Bull. Amer. Math. Soc. 39 (2002), 145-205; math.ra/0105155.

[2] S. E. Barnes. New method for the Anderson model. J. Phys. F 6 (1976), 1375-1383.

[3] M. N. Chernodub. Yang-Mills theory in Landau gauge as a liquid crystal. Phys. Lett. B 637 (2006), 128-132.

[4] P. Coleman. New approach to the mixed-valence problem. Phys. Rev. B 29 (1984), 3035-3044.

[5] V. Dzhunushaliev. Hamilton's equations in a non-associative quantum theory; hep-th/0602047.

[6] V. Dzhunushaliev. White operators in a non-associative quantum theory. Phys. Lett. A 355 (2006), 298-302; hep-th/0507032.

[7] L.D. Faddeev and A.J. Niemi. Aspects of Electric magnetic duality in SU(2) Yang-Mills theory. Phys. Lett. B 525 (2002), 195-200.

[8] P. A. Lee and N. Nagaosa. Ginzburg-Landau theory of the spin-charge-separated system. Phys. Rev. B 45 (1992), 966-970.

[9] P.A. Lee, N. Nagaosa, and X.-G. Wen. Doping a Mott insulator: physics of High Temperature superconductivity. Rev. Mod. Phys. 78 (2006), 17-86; cond-mat/0410445.

[10] J. Lõhmus, E. Paal, and L. Sorgsepp. Nonassociative Algebras in Physics. Hadronic Press, Palm Harbor, 1994.

[11] J. Lõhmus, E. Paal, and L. Sorgsepp. About Nonassociativity in Mathematics and Physics. Acta Appl. Math. 50 (1998), 3-31.

[12] A. J. Niemi and N. R. Walet. Splitting the gluon? Phys. Rev. D 72 (2005), 054007-054019.

[13] A.J. Niemi. Dual superconductors and SU(2) Yang-Mills. J. High Energy Phys. 0408 (2004), 035.

[14] S. Okubo. Introduction to Octonion and Other Non-Associative Algebras in Physics. Cambridge University Press, Cambridge, 1995. 